\title{
Avaliação antropométrica e do ângulo quadricipital na osteoartrite de joelho em mulheres obesas
}

\author{
Anthropometric and $Q$ angle assessment in obese women with knee osteoarthritis
}

\author{
Eduardo Paul Chacur', Luciana Oliveira e Silva², Gabriela Costa Pontes Luz², \\ Fábio Diodi Kaminice ${ }^{2}$ Nadia Carla Cheik ${ }^{3}$
}

\author{
Estudo desenvolvido no \\ Programa de Mestrado em \\ Fisioterapia do Unitri - Centro \\ Universitário do Triângulo, \\ Uberlândia, MG, Brasil \\ 1 Fisioterapeuta; Prof. Ms. do \\ Unitri \\ 2 Fisioterapeutas \\ 3 Profa. Dra. adjunta da \\ Faculdade de Educação Física \\ da Universidade Federal de \\ Uberlândia

\section{ENDEREÇO PARA} \\ CORRESPONDÊNCIA \\ Nadia C. Cheik \\ Faculdade de Educação Física / \\ UFU \\ R. Benjamin Constant 1286 \\ Bairro Aparecida \\ 38400-678 Uberlândia MG \\ e-mail: nadiacheik@terra.com.br; \\ eduardopc@unipam.edu.br; \\ luciana.fisioterapia@gmail.com; \\ gabpontes@hotmail.com; \\ fkaminice@hotmail.com
}

\section{ApresentaÇÃo} set. 2009

ACEITO PARA PUBLICAÇÃO maio 2010
Resumo: A osteoartrite (OA) é uma doença articular degenerativa, caracterizada por processo inflamatório, dor e deformidades; um de seus fatores preditivos é a obesidade. O objetivo deste estudo foi verificar possíveis correlações entre medidas antropométricas, o ângulo quadricipital (Q) e a osteoartrite de joelho. A amostra foi composta por 50 voluntárias obesas (30 com OA de joelho e 20 sem OA), com idade entre 40 e 60 anos. Foram mensurados, além do IMC (índice de massa corporal), circunferência abdominal (CA), perímetros de cintura e quadril para cálculo da relação cintura-quadril e o ângulo Q; a osteoartrite foi diagnosticada clinicamente e por meio de radiografia da articulação do joelho. Foram encontradas correlações positivas fracas entre IMC e ângulo Q e entre tempo de obesidade e grau de degeneração articular. A CA apresentou correlação positiva fraca com o grau de degeneração articular e o de gravidade da OA. O cálculo da razão de chance (OR) indica que as voluntárias com IMC>34 kg/m² e CA>110 cm tiveram 3,7 e 7 vezes, respectivamente, mais chance de apresentarem OA. A obesidade central, seu grau e duração possivelmente contribuem para a incidência da OA de joelhos em mulheres obesas. A circunferência abdominal foi a medida que melhor se correlacionou com a presença e grau de OA em obesas, o que aponta para a relevância de sua mensuração na avaliação clínica.

Descritores: Antropometria; Mulheres; Obesidade; Osteoartrite do joelho

ABSTRACT: Osteoarthritis (OA) is a degenerative joint disease characterized by inflammatory process, pain, and deformity; one of its main predictive factors is obesity. The aim of this study was to search for possible correlations between anthropometric measures, the $\mathrm{Q}$ angle and knee osteoarthritis. A sample of 50 obese women (30 with knee osteoarthritis and 20 with no joint disease), aged between 40 to 60 years, were assessed as to BMI (body mass index), abdominal circumference $(A C)$, waist and hip perimeters (so as to calculate waist-hip ratio), and the Q angle; osteoarthritis was diagnosed by clinical exam and knee joint radiography. Results showed a positive, poor correlation between BMI and Q angle, as well as between time of obesity onset and degree of joint degeneration. AC was found to positively, though weakly, correlate with the degree of joint degeneration and of OA severity. Adjusted odds ratio for OA showed that women with BMI>34 $\mathrm{kg} / \mathrm{m}^{2}$ and $\mathrm{AC}>110 \mathrm{~cm}$ were respectively 3.7 and 7 times more likely to develop $\mathrm{OA}$. The degree and duration of central obesity possibly contribute to incidence of knee OA in obese women. Abdominal circumference was the measure that most correlated with the degree of joint degeneration and of OA severity, which suggests it should be used in clinical evaluation.

KeY words: Anthropometry; Obesity; Osteoarthritis, knee; Women 


\section{INTRODUÇÃO}

A osteoartrite (OA) é uma doença degenerativa que atinge as articulações sinoviais, provocando degeneração da cartilagem articular, alterações inflamatórias, dor, deformidades, comprometimento da marcha e da funcionalidade nas atividades de vida diária; apresenta como um dos principais fatores de risco a obesidade ${ }^{1,2}$. No Brasil, a prevalência de osteoartrite é de 16,9\%, responsável por 30 a $40 \%$ das consultas em ambulatórios de reumatologia e por 7,5\% de todos os afastamentos de trabalho, sendo a quarta doença a determinar aposentadoria $(6,2 \%)^{3}$.

Estudos clínicos e radiológicos mostram que a incidência da osteoartrite aumenta de forma significativa entre a quarta e a quinta década de vida, no período da menopausa em mulheres e a partir dos 50 anos nos homens, afetando $60 \%$ das pessoas com 65 anos ou mais e $80 \%$ daquelas com 75 anos ou mais $^{4}$, sendo a articulação do joelho a mais acometida ${ }^{5}$. A OA do joelho contribui de forma significativa para incapacidade funcional ${ }^{6,7}$.

A relação entre OA e obesidade é explicada por fatores genéticos, metabólicos, endócrinos e mecânicos ${ }^{3}$. A quantidade e a distribuição de gordura corporal também podem estar relacionadas com a OA, variáveis estas que podem ser facilmente quantificadas por meio de avaliações antropométricas indiretas, como a razão cintura-quadril (RCQ) e a circunferência abdominal (CA). Essas mensurações são procedimentos de baixo custo e alta aplicabilidade $^{4}$, além de serem classicamente utilizadas para determinar a relação entre obesidade e doenças crônico-degenerativas $^{1}$. O excesso de sustentação de peso em um compartimento do joelho induz à degeneração da cartilagem e à OA precoce, prejudicando o alinhamento do joelho conforme aumenta a destruição da cartilagem ${ }^{8}$ - o que aponta para a importância de avaliar o ângulo quadricipital (ângulo Q) nessa população.

As modificações antropométricas e metabólicas relacionadas à obesidade podem influenciar a gravidade da OA. Assim, o objetivo deste estudo foi verificar possíveis correlações entre características antropométricas, ângulo Q e osteoartrite de joelho em mulheres obesas.

\section{METODOLOGIA}

O estudo foi realizado após aprovação do Comitê de Ética em Pesquisa em Humanos do Centro Universitário do Triângulo. As voluntárias foram orientadas sobre as etapas e procedimentos da pesquisa e assinaram o termo de consentimento livre e esclarecido.

Para este estudo foram selecionadas 50 voluntárias (30 obesas com osteoartrite de joelho e 20 obesas sem osteoartrite), com idade entre 40 e 60 anos. As voluntárias com diagnóstico de osteoartrite de joelho procederam do Hospital de Clínicas da Universidade Federal de Uberlândia e da Clínica Escola do Centro Universitário do Triângulo; foram selecionadas aquelas com índice de massa corporal (IMC) acima de $30 \mathrm{~kg} /$ $\mathrm{m}^{2}$, bom nível de entendimento e estabilidade clínica no momento da avaliação, sendo reunidas no grupo experimental (OA). O grupo controle foi constituído por mulheres obesas sem OA indicadas pelas voluntárias do grupo experimental. Foram critérios de exclusão a presença de lesões musculoesqueléticas em membros inferiores (MMII) que não a OA de joelhos, discrepância entre membros inferiores maior que 1,5 $\mathrm{cm}$, presença de doenças imunológicas, deficit neurológico, cadeirantes, mulheres eutróficas ou que já tivessem sofrido fratura nos MMII.

O critério utilizado para o diagnóstico da obesidade foi o índice de massa corporal, resultado da divisão do peso corporal pela altura ao quadrado, conforme estabelecido pela Organização Mundial de Saúde ${ }^{9}$. A relação cinturaquadril (RCQ) e a circunferência abdominal (CA) foram mensuradas para classificar a obesidade em central ou periférica. A RCQ foi calculada pela divisão da circunferência abdominal pelo perímetro dos quadris, com as voluntárias em posição ortostática. RCQ superior a 0,8 indica distribuição de gordura central. A CA foi mensurada no ponto médio entre a última costela e a crista ilíaca superior, por meio de uma fita métrica inelástica; valores abaixo de $80 \mathrm{~cm}$ foram considera- dos normais; superiores a $80 \mathrm{~cm}$ caracterizam aumento da CA, que é um indicador de obesidade central ${ }^{10}$.

Após esses procedimentos, mediu-se o ângulo Q, posicionando o eixo do goniômetro no centro da patela, com o braço fixo ao longo do fêmur em direção à espinha ilíaca ântero-superior e o braço móvel na tuberosidade tibial. Foi considerado fisiológico o ângulo quadricipital entre $14^{\circ}$ e $20^{\circ}$ : acima desse valor considerou-se a presença de valgismo e, abaixo de $14^{\circ}$, varismo ${ }^{11}$.

O diagnóstico de osteoartrite foi obtido clinicamente e por meio de radiografia em perfil e ântero-posterior da articulação do joelho, realizada por dois ortopedistas que, com vasta experiência clinica, classificaram o grau de osteoartrite de acordo com a escala de Kellgren e Lawrence ${ }^{12}$. Nessa classificação os graus de comprometimento articular variam de 1 a 4: no grau 1 o indivíduo apresenta diminuição do espaço articular duvidosa e possível labiação das bordas; no grau 4 apresenta ampla osteofitose, diminuição articular bem marcada, esclerose severa das bordas articulares e deformidades ósseas angulares já bem definidas.

O índice de Lequesne, composto por 11 questões sobre dor, desconforto e função, foi usado para classificar subjetivamente a gravidade da OA de joelhos: são seis questões sobre dor e desconforto específicas para a articulação do joeIho, uma sobre distância máxima de caminhada e quatro específicas sobre atividades de vida diária. As pontuações variam de 0 a 24 (de sem acometimento a extremamente grave $)^{13}$. Foi analisado também o comprometimento funcional das obesas com osteoartrite de joelho pelo questionário de Gordon ${ }^{14}$, que classifica a osteoartrite em quatro categorias, de acordo com a capacidade do indivíduo em executar as atividades de vida diária (AVD) como autocuidado, deslocamento e atividades instrumentais (AVI).

Para avaliar o nível de atividade física habitual das voluntárias utilizou-se o questionário de Baecke et al. ${ }^{15}$ : esse instrumento fornece três escores de atividade física realizada nos últimos doze meses, incluindo atividades ocupacionais, exercícios físicos e atividades de lazer e locomoção ${ }^{16}$. 


\section{Análise estatística}

Os dados foram analisados usando o programa SPSS (v.12.0). Na análise descritiva aplicou-se o teste de KolmogorovSmirnov para verificar se os dados apresentavam distribuição normal. Para a comparação entre os grupos utilizou-se o teste de Mann-Whitney para amostras independentes; posteriormente foi usada a correlação de Spearman (r) para os dados não-paramétricos: $r<0,25$ correlação ausente; $0,25<\mathrm{r}<0,5$ fraca a moderada; $0,5<r<0,75$ moderada a boa; $r>0,75$ boa a excelente. Foi também utilizado o programa BioEstat (v.4.0) para aplicar o teste de razões de chance (odds ratio, OR). O nível de significância foi estabelecido em $p<0,05$.

\section{RESULTADOS}

O grupo osteoartrite (OA), com 30 voluntárias, tinha idade média de 52 anos; no grupo controle (GC), composto por 20 obesas sem osteoartrite, a idade média foi 48 anos (Tabela 1). As voluntárias do grupo OA apresentaram valores maiores de idade, IMC e CA $(p<0,05)$, mas não houve diferença estatisticamente significante na RCQ entre os grupos. Todas as voluntárias apresentaram circunferência abdominal aumentada e $23 \%$ apresentaram RCQ $>0,9$. Quanto ao nível de atividade física e ao ângulo quadricipital, o grupo OA apresentou menor nível de atividade física, não havendo diferenças significativas entre os grupos em relação ao ângulo quadricipital (Tabela 1). Não foi observada correlação entre os valores do ângulo Q e o grau, gravidade ou funcionalidade da OA.

As medidas antropométricas do grupo OA segundo a classificação do grau de OA são apresentadas na Tabela 2. Nesse grupo, foi encontrada correlação positiva fraca entre IMC e ângulo quadricipital (Tabela 3), sugerindo que o aumento da massa corporal está associado à alteração do ângulo quadricipital $\left(>20^{\circ}\right)$. Relembre-se que as voluntárias desse grupo foram classificadas quanto ao grau de OA (escala de Kellgren \& Lawrence), gravidade da OA (índice de Lequesne) e funcionalidade (questionário de Gordon), sendo testadas correlações
Tabela 1 Valores médios ( \pm desvio padrão) da idade, medidas antropométricas, tempo de obesidade (TO), nível de atividade física (NAF) e ângulo Q dos grupos controle (GC) e osteoartrite (OA) e valor de $p$ da comparação entre os grupos; $n=50$

Característica GC $(\mathrm{n}=20)$ OA $(\mathrm{n}=30) \quad p$ \begin{tabular}{llll}
\hline Idade (anos) & $48,0 \pm 5,7$ & $52,0 \pm 5,3$ & 0,04
\end{tabular}

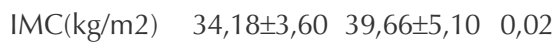
RCQ $\quad 0,87 \pm 0,07 \quad 0,88 \pm 0,06 \quad 0,55$ CA (cm) 106,4 $111,1 \quad 113,4 \pm 9,5 \quad 0,03$ TO (anos) $\quad 15,5 \pm 11,1 \quad 17,9 \pm 11,1 \quad 0,42$ NAF $\quad 4,5 \pm 0,4 \quad 3,4 \pm 0,9 \quad 0,04$ Ângulo Q (D) $\quad 18,8 \pm 3,6 \quad 19,9 \pm 4,4 \quad 0,14$ Ângulo Q (E) $\quad 18,9 \pm 4,4 \quad 19,7 \pm 4,6 \quad 0,55$ Maior ângulo Q 19,8 $\pm 3,8 \quad 21,4 \pm 4,2 \quad 0,12$ $\mathrm{IMC}=$ índice de massa corporal; RCQ = relação cintura quadril; $C A=$ circunferência abdominal; $\mathrm{D}=$ direito; $\mathrm{E}$ = esquerdo

Tabela 2 Variáveis antropométricas do grupo OA (média \pm desvio padrão) segundo o grau de osteoartrite; $n=30$

\begin{tabular}{cccc}
\hline Grau & IMC $\left(\mathrm{kg} / \mathrm{m}^{2}\right)$ & $\mathrm{CA}(\mathrm{cm})$ & RCQ \\
\hline 1 & $32,4 \pm 1,1$ & $102,0 \pm 9,0$ & $0,93 \pm 0,1$ \\
2 & $37,0 \pm 5,0$ & $112,1 \pm 9,6$ & $0,89 \pm 0,1$ \\
3 & $38,2 \pm 5,5$ & $116,1 \pm 8,6$ & $0,88 \pm 0,1$ \\
4 & $37,5 \pm 0,1$ & $117,0 \pm 0,1$ & $0,90 \pm 0,1$ \\
\hline
\end{tabular}

$\mathrm{IMC}=$ índice de massa corporal; $\mathrm{RCQ}=$ relação cintura quadril; $C A$ = circunferência abdominal

Tabela 3 Correlações (r) das variáveis antropométricas com grau de OA e ângulo Q no grupo OA

\begin{tabular}{lcc}
\hline Variável & Grau de OA & Ângulo Q \\
\hline IMC $\left(\mathrm{kg} / \mathrm{m}^{2}\right)$ & 0,12 & $0,44 *$ \\
RCQ & $-0,09$ & 0,08 \\
CA $(\mathrm{cm})$ & $0,38 *$ & 0,23 \\
TO (anos) & $0,44 *$ & 0,00 \\
\hline
\end{tabular}

$\mathrm{IMC}=$ índice de massa corporal; $\mathrm{RCQ}=$ relação cintura quadril; $\mathrm{CA}=$ circunferência abdominal; TO = tempo de obesidade; * $p<0,05$

Tabela 4 Prevalência e razão de chance (OR, intervalo de confiança IC 95\%) segundo

valores selecionados das medidas antropométricas, tempo de obesidade (TO) e ângulo Q, nos grupos controle (GC) e com osteoartrite (OA)

\begin{tabular}{|c|c|c|c|c|}
\hline \multirow{2}{*}{ Variável } & \multicolumn{2}{|c|}{ Prevalência (\%) } & \multicolumn{2}{|c|}{ Razão de chance } \\
\hline & GC & $\mathrm{OA}$ & GC & $\mathrm{OA}$ \\
\hline $\mathrm{CA}>110 \mathrm{~cm}$ & 30 & 70 & $0,14(0,03-0,51) *$ & $7,00(1,94-25,13) *$ \\
\hline $\mathrm{RCQ}>0,88$ & 45 & 30 & $1,90(0,58-6,19)$ & $0,52(0,16-1,69)$ \\
\hline $\mathrm{TO} \geq 5$ anos & 30 & 18 & $0,72(0,11-4,37)$ & $1,38(0,22-8,38)$ \\
\hline Ângulo $\mathrm{Q}>20^{\circ}$ & $\circ 60$ & 76,6 & $0,45(0,13-1,56)$ & $2,19(0,63-7,50)$ \\
\hline $\mathrm{IMC}>34$ & 35 & 66,6 & $0,26(0,08-0,81)$ & $3,71(1,12-12,2) *$ \\
\hline
\end{tabular}

$\mathrm{IMC}=$ índice de massa corporal; $\mathrm{RCQ}=$ relação cintura quadril; $\mathrm{CA}=$ circunferência abdominal * $p<0,05$

entre as variáveis antropométricas, tempo de obesidade (TO) e essas classificações.

Correlações positivas fracas foram encontradas do grau de OA com CA e TO (Tabela 3). Ao serem testadas correlações das variáveis IMC, RCQ e TO com gravidade, funcionalidade da $\mathrm{OA}$ e o nível de atividade física, não foram encontrados dados significantes; apenas a CA correlacionou-se fracamente $(r=0,27$; $p<0,05)$ com a gravidade de OA. Após análise da razão de chance, observouse que as voluntárias com IMC>34 kg/ $\mathrm{m}^{2}$ e CA $>110 \mathrm{~cm}$ tiveram 3,7 e 7 vezes respectivamente mais chance de apresentarem OA (Tabela 4).

\section{DISCUSSÃO E CONCLUSÃO}

A pertença ao sexo feminino e a idade crescente são considerados fatores de risco para a OA. Além disso, estudos mostram que a maior prevalência de sobrepeso e obesidade resultam em alta incidência da OA de joelho nessa população ${ }^{12,13,17}$. Justifica-se ainda a amostra feminina do presente estudo pelo fato de os sintomas da OA ocorrerem antecipadamente em mulheres ${ }^{17}$. Ismail et al. ${ }^{18}$, examinando mais de 200 pacientes com OA de joelhos, encontraram que mais de $90 \%$ eram obesos ou com sobrepeso, sendo maior a prevalência no sexo feminino, em uma relação de 2,4:1 para o sexo masculino. A maior idade das voluntárias do grupo OA em relação às do GC pode ser um fator de confusão. Embora na análise intragrupo tenha-se observado que a idade apresentou correlação positiva somente com o grau da OA, teria sido importante parear os grupos por idade. Entretanto, como o grupo controle

(n)


foi recrutado dentro do convívio do grupo com OA, foram priorizados outros fatores de risco para a OA de joelho.

A obesidade é conhecida como um fator de risco para inúmeras doenças crônicas que têm como base a inflamação sistêmica. Evidências indicam que a obesidade tem repercussão negativa em estruturas como tendões, fáscias $e$ cartilagens ${ }^{19}$, o que pode estar associado às alterações antropométricas. A maior prevalência de OA de joelho em mulheres e sua correlação com o aumento do IMC ${ }^{20,21}$ corroboram os resultados do presente estudo, onde mulheres com IMC> $34 \mathrm{~kg} / \mathrm{m}^{2}$ apresentaram chance 3,7 vezes maior de desenvolverem $\mathrm{OA}^{22-24}$.

Estudos mostram que, além do IMC, a CA também apresenta correlação positiva com a $\mathrm{OA}^{10,19}$, o que corrobora nossos resultados. Apesar de a correlação encontrada ter sido fraca, possivelmente devido ao tamanho da amostra, a análise de razão de chance indicou que as mulheres do grupo OA com CA $>110 \mathrm{~cm}$ apresentavam 7 vezes mais chance de serem acometidas por OA de joelhos. Também foi observado que todas as voluntárias do grupo OA apresentaram CA aumentada, indicando aumento do risco de complicações associadas à obesidade central. Uma das hipóteses que poderia explicar a ligação da OA com a obesidade, especialmente a obesidade central, é a de que fatores metabólicos e circulantes relacionados com o tecido adiposo visceral afetariam adversamente a cartilagem e outras estruturas articulares, favorecendo assim o desenvolvimento da $\mathrm{OA}^{25}$; tais fatores pró-inflamatórios poderiam ser fator de necrose tumoral alfa (TNF-a), proteína $C$ reativa (PCR) e interleucinas (IL-1, IL-
6, IL-17) ${ }^{25,26}$. A IL-1, uma citocina próinflamatória importante na instalação do foco inflamatório, tem a função de induzir os condrócitos e os sinoviócitos a produzirem as metaloproteases e prostaglandinas, além de deprimir a síntese do colágeno tipo II e dos proteoglicanos (reparadores da cartilagem) e as somatomedinas que impedem a proliferação dos condrócitos. A IL-1 também aumenta a produção do óxido-nítrico, o qual induz o desarranjo estrutural dos condrócitos $^{27}$. Concomitantemente, a liberação aumentada de TNF-a e IL-6 pelo tecido adiposo central resulta em elevados níveis circulantes de PCR e de fibrinogênio. O aumento da PCR tem sido associado à osteoartrite de joelho no sexo feminino ${ }^{26}$. Ainda, alguns achados sugerem que níveis elevados de TNFa plasmático contribuem para a susceptibilidade aumentada à inflamação $0^{28,29}$. Uma das limitações do presente estudo é, diante dos achados antropométricos, não ter efetuado a dosagem de citocinas pró-inflamatórias.

Além de fatores metabólicos, fatores mecânicos também estão relacionados à osteoartrite. Sharma et al. ${ }^{30}$ afirmam que a rápida progressão da OA de joeIho, devido ao mau alinhamento, predispõe ao declínio na função física. Neste estudo esse achado é confirmado, pois o nível de atividade física do grupo OA foi inferior ao do GC e, pelo questionário Gordon, pôde-se observar que $80 \%$ do grupo OA relataram dificuldade nas atividades recreativas e esportivas.

O ângulo quadricipital excessivo (valgismo) aumenta o contato lateral da articulação fêmuro-patelar ${ }^{31}$ e proporciona melhor tolerância à sobrecarga articular imposta pela obesidade, quando comparado ao varismo ${ }^{30}$; isso justifica a alta prevalência de valgismo (83\%) neste estudo. $\mathrm{O}$ excesso de sustentação de peso em um compartimento do joelho induz à degeneração da cartilagem e à OA precoce, e o alinhamento piora à medida que aumenta a destruição da cartilagem $^{32}$.

Possivelmente o maior tempo de obesidade e circunferência abdominal aumentada tenham influenciado o grau de comprometimento da OA. Para avaliar o grau da OA foi realizada análise radiográfica da articulação do joelho. Entretanto, o diagnóstico clínico da radiografia nem sempre se coaduna com a sintomatologia ${ }^{32}$. Assim, foi de grande valia a aplicação do questionário Lequesne, que contempla dor, disfunção da marcha e AVD para avaliação do estadiamento da doença ${ }^{32}$. Observou-se que as voluntárias que apresentavam OA muito grave $(37 \%)$ possuíam maior CA e IMC (dados não apresentados). Sugere-se pois que os questionários que avaliam a gravidade da OA e o comprometimento da funcionalidade devam ser utilizados no diagnóstico e na estimativa do risco de progressão da OA de joeIho em mulheres obesas.

Estes achados confirmam que a obesidade, principalmente central, está relacionada à OA, o que resulta em redução do nível de atividade física e pode contribuir para o estabelecimento de ciclo vicioso entre obesidade, OA e sedentarismo. Foi observada fraca correlação entre o IMC e o ângulo Q. A circunferência abdominal foi a variável antropométrica que melhor se correlacionou com a presença e grau de OA. Assim, destaca-se a importância da mensuração da CA na avaliação clínica de pacientes com osteoartrite de joelho.

\section{REFERÊNCIAS}

1 Botha-Scheepers S, Watt I, Rosendaal FR, Breedveld FC, Hellio-le-Graverand MP, Kloppenburg M. Changes in outcome measures for impairment, activity limitation, and participation restriction over two years in osteoarthritis of the lower extremities. Arthritis Rheum. 2008;59(12):1750-5.
2 Das SK, Farooqi A. Osteoarthritis. Best Pract Res Clin Rheumatol. 2008;22(4):657-75.

3 Carvalho MAP, Lanna CCD, Bértolo MB. Reumatologia: diagnóstico e tratamento. Rio de Janeiro: Guanabara Koogan; 2008. p.245-53. 

Gamia. Rio de janeiro: Rubio; 2008.

5 Michael JW, Schlüter-Brust KU, Eysel P. The epidemiology, etyology, diagnosis, and treatment of osteoarthritis of the knee. Dtsch Arztebl Int. 2010;107(9):152-62.

6 Sonne-Holm S, Jacobsen S. Osteoarthritis and obesity. Ugeskr Laeger. 2006;168(2):187-90.

7 Hajian-Tilaki KO, Heidari B. Prevalence of obesity, central obesity and the associated factors in urban population aged 20-70 years, in the North of Iran: a population-based study and regression approach. Obes Rev. 2007;8(1):3-10.

8 Ferraz MB, Ciconelli RM. Artrite reumatóide. In: Borges DR, Rothschild HA, organizadores. Atualização terapêutica. 20a ed. São Paulo: Artes Médicas; 2001. p.1378-82.

9 World Health Organization. Physical status: the use and interpretation of anthropometry. Geneva; 1995. (Technical Report Series, 854)

10 Janssen I, Mark AE. Separate and combined influence of body mass index and waist circumference on arthritis and knee osteoarthritis. Int J Obes. 2006;30:1223-8.

11 Tribastone F. Tratado de exercícios corretivos aplicados à reeducação motora postural. São Paulo: Manole; 2005.

12 Kellgren JH, Lawrence JS. Radiological assessment of osteoarhtritis. Ann Rheum Dis. 1957;16:494-502.

13 Marx FC, Oliveira LM, Bellini CG, Ribeiro MCC. Tradução e validação cultural do questionário algofuncional de Lequesne para osteoartrite de joelhos e quadris para a língua portuguesa. Rev Bras Reumatol. 2006;46(4):253-60.

14 Gordon NF. Arthritis: your complete exercise guide: the Cooper Clinic and Research Institute fitness series. Dallas, TX: Human Kinetics; 1993.

15 Baecke JAH, Burema J, Frijters JER. A short questionnaire for the measurement of habitual physical activity in epidemiological studies. Am J Clin Nutr. 1982;36:936-42.

16 Florindo AA, Latorre MR, Jaime PC, Tanaka T, Zerbini CAF. Metodologia para a avaliação da atividade física habitual em homens com 50 anos ou mais. Rev Saude Publ. 2004;38(2):307-14.

17 Grotle M, Hagen KB, Natvig B, Dahl FA, Kvien TK. Obesity and ostheoarthritis in knee, hip and/or hand: an epidemiological study in the general population with 10 years follow-up. BMC Musculoskelet Disord. 2008;9:132.

18 Ismail Al, Al-Abdulwahab AH, Al-Mulhim AS. Osteoarthritis of knees and obesity in Eastern Saudi Arabia. Med Saudi J. 2006;27(11):1742-4.
19 Abbate LM, Stevens J, Schwartz TA, Renner JB, Helmick CG, Jordan JM. Anthropometric measures, body composition, body fat distribution, and knee osteoarthritis in women. Obesity. 2006;14:1274-81.

20 Szoeke, CEI, Cicuttini FM, Guthrie JR, Clark MS, Dennerstein L. Factors affecting the prevalence of osteoarthritis in healthy middle-aged women: data from the longitudinal Melbourne Women's Midlife Health Project. Bone. 2006;39(5):1149-55.

21 Segal NA, Yack HJ, Khole P. Weight, rather than obesity distribution, explains peak external knee adduction moment during level gait. Am J Phys Med Rehabil. 2009;88(3):189-91.

22 Marks R. Obesity profiles with knee osteoarthritis: correlation with pain, disability, disease progression. Obesity. 2007;15(7):1867-74.

23 Jinks C, Jordan K, Croft P. Disabling knee pain - another consequence of obesity: results from a prospective cohort study. BMC Public Health. 2006;19(6):25-8

24 Grazio S, Balen D. Obesity: risk factor and predictor of osteoarthritis [abstract]. Lijec Vjesn. 2009;131(1-2):22-6.

25 Blom AB, Van der Kraan PM, Van der Berg WB. Cytokine targeting in osteoarthritis. Curr Drug Targets. 2007;8(2):283-92.

26 Engström G, Gerhardsson de Verdier M, Rollof J, Nilsson PM, Lohmander LS. C-reactive protein, metabolic syndrome and incidence of severe hip and knee osteoarthritis: a population-based cohort study. Osteoarthritis Cartilage. 2009;17(2):168-73.

27 Bonsderson J, Wainwright S, Hughes C, Caterson B. The regulation of the ADAMTS4 and ADAMTS5 aggrecanases in osteoarthritis: a review. Clin Exp Rheumatol. 2008;26:1:139-45.

28 Calich AL, Domiciano DS, Fuller R. Osteoarthritis: can anti-cytokine therapy play a role in treatment? Clin Rheumatol. 2010;29(5):451-5.

29 Lai PP, Leung AK,Li AN, Zhang M. Three-dimensional gait analysis of obese adults. Clin Biomech (Bristol, Avon). 2008;23(Suppl 1):S2-6.

30 Sharma L, Kapoor D, Issa S. Epidemiology of osteoarthritis: an update. Cur Opin Rheumatol. 2006;18(2):147-56.

31 Mizuno Y, Kumagai M, Mattessich SM, Elias JJ, Ramrattan N, Cosgarea AJ, et al. Q-angle influences tibiofemoral an patellofemoral kinematcs. J Orthop Res. 2001;19:834-40.

32 Bedson J, Croft PR. The discordance between clinical and radiographic knee osteoarthritis: a systematic search and summary of the literature. BMC Musculoskelet Disord. 2008;2;9:116. 